\title{
Antigenic analysis of grass carp reovirus using single-chain variable fragment antibody against IgM from Ctenopharyngodon idella
}

\author{
CHEN CongLin ${ }^{1}$, SUN XiaoYun ${ }^{2}$, LIAO LanJie ${ }^{1}$, LUO ShaoXiang ${ }^{1}$, LI ZhouQuan ${ }^{1}$, \\ ZHANG XiaoHua ${ }^{1}$, WANG YaPing ${ }^{1}$, GUO QionLin ${ }^{1}$, \\ ${\text { FANG } \text { Qin }^{2 *} \& \text { DAI HePing }}^{1 *}$ \\ ${ }^{1}$ State Key Laboratory of Freshwater Ecology and Biotechnology, Institute of Hydrobiology, Chinese Academy of Sciences, \\ Wuhan 430072, China; \\ ${ }^{2}$ State Key Laboratory of Virology, Wuhan Institute of Virology, Chinese Academy of Sciences, Wuhan 430071, China
}

Received August 28, 2012; accepted November 28, 2012

\begin{abstract}
Grass carp (Ctenopharyngodon idella) is an important species of freshwater aquaculture fish in China. However, grass carp reovirus (GCRV) can cause fatal hemorrhagic disease in yearling populations. Until now, a strategy to define the antigenic capacity of the virus's structural proteins for preparing an effective vaccine has not been available. In this study, some single-chain variable fragment antibodies ( $\mathrm{scFv}$ ), which could specifically recognize grass carp IgM, were selected from a constructed mouse naïve antibody phage display cDNA library. The identified scFv C1B3 clone was shown to possess relatively higher specific binding activity to grass carp IgM. Furthermore, ELISA analysis indicated that the IgM level in serum from virus-infected grass carp was more than two times higher than that of the control group at 5-7 days post infection. Moreover, Western blot analysis demonstrated that the outer capsid protein VP7 has a specific immuno-binding-reaction with the serum IgM from virus-infected grass carp. Our results suggest that VP7 can induce a stronger immune response in grass carp than the other GCRV structural proteins, which implies that VP7 protein could be used as a preferred immunogen for vaccine design.
\end{abstract}

grass carp, grass carp reovirus (GCRV), IgM, single-chain variable fragment (scFv), antigenicity

Citation: Chen C L, Sun X Y, Liao L J, et al. Antigenic analysis of grass carp reovirus using single-chain variable fragment antibody against IgM from Ctenopharyngodon idella. Sci China Life Sci, 2013, 56: 59-65, doi: 10.1007/s11427-012-4425-5

Grass carp (Ctenopharyngodon idella) is one of the most important freshwater aquatic animals in China. However, grass carp reovirus (GCRV), a fatal pathogen to aquatic animals, can provoke severe hemorrhagic disease in fingerling and yearling populations of grass carp, and cause a mortality rate of up to $85 \%$ during an outbreak [1-4]. Due to the high virulence of GCRV, the production of grass carp is severely affected and large economic losses in freshwater aquaculture in China have occurred since the 1980s [5]. GCRV also infects black carp (Mylopharyngodon piceus),

*Corresponding author (email: qfang@wh.iov.cn; hpdai@ihb.ac.cn) topmouth gudgeon (Pseudorasbora parva) and rare minnow (Gobiocypris rarus) [6,7]. Notably, GCRV was recognized to be the most virulent agent amongst all of the identified aquareovirus isolates in the genus Aquareovirus of the Reoviridae family [8]. In an attempt to control the spread of the disease, several inactive vaccines have been developed over the years. However, epidemic outbreaks of the hemorrhagic disease have still occurred in many freshwater culture areas in recent years. To better mitigate the disease, it is necessary to characterize the antigenicity of GCRV in grass carp to develop a novel vaccine against it.

GCRV has been assigned to the genus Aquareovirus in 
the family Reoviridae. Similar to other reoviruses, GCRV is a non-enveloped icosahedral particle about $80 \mathrm{~nm}$ in diameter. It consists of an eleven segmented double-stranded RNA genome enclosed by two concentric icosahedral protein capsids, which consist of seven structural proteins VP1-VP7. The inner core layer is arranged with $\mathrm{T}=1$ symmetry, is composed of five proteins, and possesses the enzymatic activities necessary for viral transcription and replication [9-12]. The remaining VP5 and VP7 proteins are outer capsid proteins arranged on an incomplete $\mathrm{T}=13$ icosahedral lattice, which contains 200 trimers formed by VP5-VP7 heterodimers. Recently, antibodies against the outer-capsid VP5 and VP7 proteins were produced from mammalian animals and evaluated in vitro by a plaque reduction neutralization assay [13-15]. To understand which protein of GCRV is highly immunogenic, the antigenic capacity of the virus structural proteins needs to be investigated in grass carp.

In lower vertebrates, immunoglobulins (Igs) have been identified and characterized in all jawed fish species, including teleost fish. Several fish Ig isotypes have been reported in teleost fish, namely $\operatorname{IgM}, \operatorname{IgD}, \operatorname{IgZ}, \operatorname{IgT}$, the $\operatorname{IgM}-\operatorname{IgD}$ chimera and the $\operatorname{IgM}-\operatorname{IgZ}$ chimera [16-19], of which $\operatorname{IgM}$ is the major isotype. Similar to mammalian IgM, each monomer of teleost IgM is composed of two heavy chains and two light chains linked by disulfide bridges. IgM is commonly a tetrameric molecule with a molecular weight between $610-900 \mathrm{kD}$. The heavy chain varies between $70-81 \mathrm{kD}$, and the light chain between $22-32 \mathrm{kD}$ in teleosts. Noncovalent bonding is a frequent feature of the association of monomers to form a complete tetramer [20]. As one of the most important antibodies against pathogens in teleost fishes, including grass carp, IgM is the primary immunoglobulin mediating humoral adaptive immunity in fish [21]. Thus, IgM can be used as a marker for humoral immune responses in fish and can also be used as a tool to identify and select specific antigens for vaccines.

To evaluate the antigenicity of GCRV in grass carp, a mouse naïve antibody (in scFv format) library displayed on phage was successfully constructed and applied for affinity selection of an $\mathrm{scFv}$ antibody against $\operatorname{IgM}$ in this study. Based on its specific binding activity to IgM in grass carp, the $\mathrm{scFv}$ C1B3 antibody was used to detect serum IgM level changes in GCRV-infected grass carp. Moreover, an immunoblotting assay demonstrated that the outer capsid protein VP7 has stronger antigenicity to elicit specific IgM production than the other structural proteins, VP1 to VP6. The results of this study indicate that VP7 is the most antigenic viral protein in grass carp.

\section{Materials and methods}

\subsection{Isolation and purification of IgM from grass carp}

IgM was isolated and purified from grass carp according to the method reported by Chen et al. [22]. Briefly, grass carp bought from a local market were bled from the tail vessel. After being clotted, the blood was centrifuged at $2000 \times \mathrm{g}$ for $15 \mathrm{~min}$ at $4^{\circ} \mathrm{C}$ to separate the serum. Afterwards, all supernatant was taken and precipitated with $30 \%-50 \%$ saturated ammonium sulfate solution, and the pellet was redissolved in $50 \mathrm{mmol} \mathrm{L}^{-1}$ Tris- $\mathrm{HCl}(\mathrm{pH} \mathrm{8.0)}$ and dialyzed against 20 mmol L ${ }^{-1}$ Tris- $\mathrm{HCl}(\mathrm{pH} 8.0$ ) for $36 \mathrm{~h}$. An affinity chromatography column with $1.4 \mathrm{~mL}$ Protein A-Sepharose 4B Fast Flow (Sigma-Aldrich, St. Louis, MO, USA) was used for affinity purification of IgM following the manufacturer's instructions. The protein concentration of purified $\operatorname{IgM}$ was estimated by the Bradford method [23] and analyzed by SDS-PAGE.

\subsection{Construction of mouse naïve antibody phage dis- play cDNA library}

To construct a mouse naïve antibody phage display cDNA library, total RNA was extracted from bone marrow stem cells, peripheral blood lymphocytes and spleens of 100 six-week-old SPF BALB/c mice using Trizol reagent (Novagen, Darmstadt, Germany). The mRNA was purified and first strand cDNA was synthesized using a First Strand cDNA Synthesis Kit (Promega, Madison, USA). The VH and VL genes were then amplified from the cDNA and assembled into the $\mathrm{scFv}$ format using a flexible linker, cloned into the phagemid vector pCANTAB $5 \mathrm{E}$ (Pharmacia, UK), and further transformed into E. coli NM522 cells as previously reported [24]. Colonies were then collected, mixed with glycerol, and stored at $-80^{\circ} \mathrm{C}$ for further use.

\subsection{Panning and identifying specific scFv antibodies against grass carp IgM}

The phage display library with $\mathrm{scFv}$ phagemid constructed above was rescued by superinfection with M13K07 helper phage. Three rounds of panning against purified IgM coated on Nunc immuno test tubes (Inter Med) were performed. According to the procedure described by Amersham Biosciences Expression Module (Amersham Biosciences Inc, $\mathrm{UK}$ ), the bound phages were eluted, infected into E. coli TG1 cells and rescued for the next round of panning. Positive clones were then identified and induced to express soluble $\mathrm{scFv}$ for specific binding assays. Each expressed $\mathrm{scFv}$ from the phage display system had an E-tag for detection.

\subsection{SDS-PAGE, Western blot and ELISA}

All samples used for protein analysis in this study were subjected to $10 \%$ or $12 \%$ sodium dodecylsulfate-polyacrylamide gel electrophoresis (SDS-PAGE) following an established method [15]. The protein samples were detected either by staining with Coomassie brilliant blue R-250 
(Sigma-Aldrich, St. Louis, MO, USA) or by transferring them to a polyvinylidene fluoride (PVDF) transfer membrane using a semi-dry transfer cell (Bio-Rad, California, USA) and visualizing by Western blotting. For the Western blotting, the membrane was blocked with 4\% PBSM (4\% skim milk in PBS) and incubated at $30^{\circ} \mathrm{C}$ for $1 \mathrm{~h}$ with grass carp serum (1:500 dilution in 4\% PBSM) either from GCRV-infected grass carp or uninfected controls. Soluble scFv C1B3 (1:8 dilution in 4\% PBSM) against grass carp IgM was used as the secondary antibody. After the membrane was washed three times with PBST $(0.1 \%$ Tween 20 in PBS) and three times with PBS, the bound scFv was detected by anti-E-tag mouse monoclonal antibody conjugated with HRP (1:10000 dilution in 4\% PBSM, Amersham Biosciences, UK) at $30^{\circ} \mathrm{C}$ for $1 \mathrm{~h}$. The color reaction was developed in substrate buffer with the 3,3-diaminobenzidine (DAB, Amresco, USA).

For ELISA, 96-well plates were coated with $200 \mathrm{ng}$ of the purified IgM in $100 \mu \mathrm{L}$ PBS per well at $4^{\circ} \mathrm{C}$ overnight for selection of positive scFv clones, or coated with $1 \mu \mathrm{L}$ of serum from grass carp in $100 \mu \mathrm{L}$ PBS for detection of IgM level changes in response to GCRV infection. After all of the coated wells were blocked with $4 \%$ PBSM, soluble scFv antibody was loaded into the plate wells and incubated at $30^{\circ} \mathrm{C}$ for $1 \mathrm{~h}$. Anti-E-tag mouse monoclonal antibody with HRP conjugate (1:10000 dilution in 4\% PBSM, Amersham Biosciences) was then added and incubated at $30^{\circ} \mathrm{C}$ for $1 \mathrm{~h}$. Finally, 3,3',5,5'-tetramethylbenzidine (Serva, Heidelberg, Germany) was used for the color reaction, which was stopped with $\mathrm{H}_{2} \mathrm{SO}_{4}$. The absorbance was determined at 450 $\mathrm{nm}$ using a spectrophotometer (BioTek, Seattle, WA, USA).

\subsection{DNA sequencing}

The genes of positive scFv clones were sequenced by the Huada Company, Shanghai, China. The primers S1 (5'CAACGTGAAAAAATTATTATTCGC-3') or S6 (5'-GTAAATGAATTTTCTGTATGAGG-3') were used for DNA sequencing.

\subsection{GCRV infection and IgM detection of immune re- sponse in grass carp}

The GCRV samples used in this study were collected and isolated with typical hemorrhagic diseased grass carp from the freshwater aquaculture farm of the Zhongbo Biological Technology Company, Wuhan, Hubei, China. The diseased fish tissues were excised, ground with three times their volume of physiological saline $(0.7 \% \mathrm{NaCl})$ to make a homogeneous suspension at $4^{\circ} \mathrm{C}$ and centrifuged for $30 \mathrm{~min}$ at $2000 \times g$. The supernatant was prepared for further detection as diseased fish tissue homogenate virus stock.

To test the changes in IgM level in response to GCRV infection, 50 yearling grass carp (from the company above) were artificially infected with $1 \mathrm{~mL}$ of GCRV homogenate supernatant per $100 \mathrm{~g}$ of weight by injection into muscle tissue and 50 yearling carp were injected with $0.7 \% \mathrm{NaCl}$ as a control group; both injections contained $100 \mathrm{U} \mathrm{mL}^{-1}$ double antibiotic (penicillin and streptomycin), as described by Wang et al. [7]. Three infected and three uninfected control fingerling carp were tail bled every day post infection (dpi) until $7 \mathrm{dpi}$; The IgM level from both the infected group and the uninfected control group were detected using $\mathrm{scFv}$ antibody against IgM from grass carp by ELISA and western blot assays as described above. Photographs were taken of the hemorrhagic diseased grass carp from the GCRV infected group and the normal grass carp from the uninfected control group.

\subsection{Purification of GCRV virions and TEM}

GCRV particles were purified as described previously [25]. Briefly, GCRV particles were primarily extracted from diseased fish tissue or cell cultured virus supernatant by different centrifugations. A low speed centrifugation at $8000 \times g$ for $20 \mathrm{~min}$ at $4^{\circ} \mathrm{C}$ was used to remove cell pellets, followed by ultracentrifugation at $80000 \times g$ in a SW28 rotor (Beckman, Massachusetts, USA) for $2 \mathrm{~h}$ at $4^{\circ} \mathrm{C}$ to pellet the virus. Further purification was conducted using cesium chloride $(\mathrm{CsCl})$ gradients with a SW40 Ti rotor (Beckman, Massachusetts, USA) spun at $105000 \times g$ for $4 \mathrm{~h}$ at $4^{\circ} \mathrm{C}$. The intact virion band was harvested and extensively dialyzed against PBS. The purified virions were negative stained and observed with a transmission electron microscope (Hitachi 7000-FA, Tokyo, Japan).

\section{Results}

\subsection{Isolation and characterization of IgM from grass carp}

To characterize the IgM from grass carp, it was first precipitated using 30\%-50\% saturated ammonium sulfate solution, and further purified with a Protein A-Sepharose 4B column. The purified and unpurified serum samples were analyzed by SDS-PAGE as shown in Figure 1. The purified IgM subunits from grass carp serum presented two clear bands at about 75.8 and $28.2 \mathrm{kD}$, which corresponded to the heavy and light chains of $\operatorname{IgM}$, respectively [21]. In a previous investigation, the molecular weight of the purified $\operatorname{IgM}$ polymer from grass carp was estimated to be approximately $800 \mathrm{kD}$ by non-reducing SDS-PAGE analysis [21]. It was clear that the molecular weight of the purified IgM polymer from grass carp was eight times the sum of the heavy chain and light chain values, which proved that grass carp IgM was a tetramer. 


\subsection{Selection and sequence alignment of anti-IgM scFv antibodies from the phage display library}

To obtain anti-IgM scFv antibodies, a large capacity scFv antibody phage display library was constructed. According to the number of ampicillin-resistant colonies, the recombinant mouse naïve antibody phage display library was estimated to contain $1.2 \times 10^{9}$ clones. Purified IgM from grass carp was immobilized as a target during three rounds of panning with the mouse naive antibody phage display library. Some positive clones were identified by ELISA as shown in Figure 2.

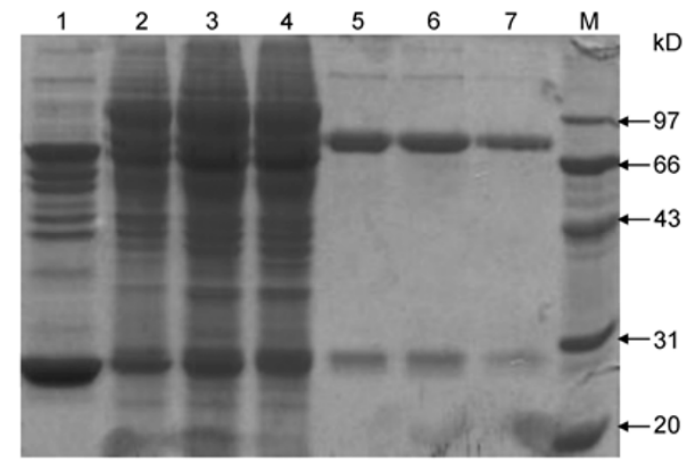

Figure 1 SDS-PAGE of the IgM from grass carp serum. Lane 1, grass carp serum; lanes 2-4, components of serum precipitated by $30 \%-50 \%$ saturated ammonium sulfate solution; lanes 5-7, IgM purified by Protein A affinity chromatography; lane $\mathrm{M}$, protein molecular weight markers.

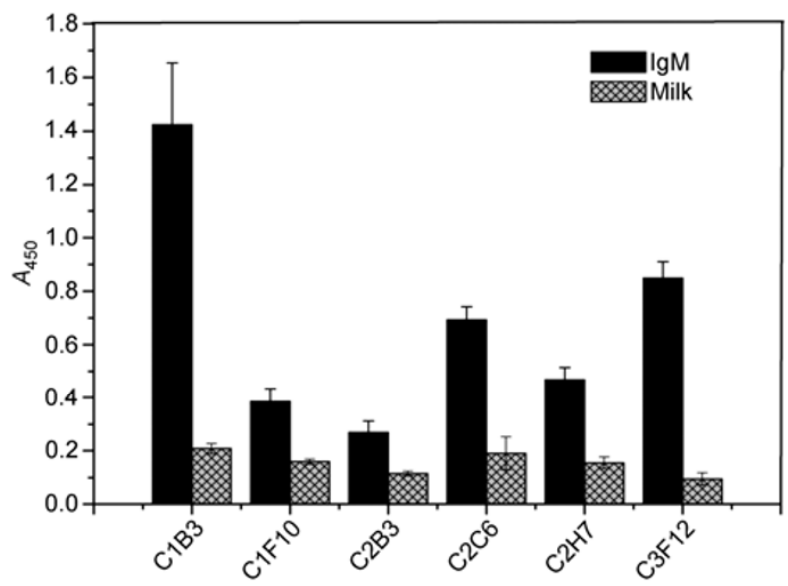

Figure 2 ELISA of positive clones from the third panning round. A 96-well plate was coated with purified IgM from carp at $200 \mathrm{ng} /$ well, with skim milk as a control. ScFvs were solubly expressed and diluted 1:2 in the assay. Values are mean $\pm \mathrm{SD}$. The asterisks denote significant difference in paired $t$ tests, $n=3, P<0.05$.

In addition, the amino acid sequences of the complementarity determining region (CDR) in the variable regions of the $\mathrm{H}$ and $\mathrm{L}$ chains of some specific scFvs were analyzed as shown in Table 1. Sequence alignment showed that scFvs from the positive clones $\mathrm{C} 1 \mathrm{~B} 3, \mathrm{C} 2 \mathrm{H} 7, \mathrm{C} 3 \mathrm{~F} 12$ were complete, and that the other scFvs from C1F10, C2B3, C2C6 had lost the variable region of the light chain. We hypothesized that the heavy chain variable region was the main part of the $\mathrm{scFv}$ binding domain to recognize IgM from grass carp. Comparing the amino acid sequences of the CDRs showed that the six scFvs were different and may bind different epitopes of IgM.

\subsection{Binding specificity assay for anti-IgM scFv anti- bodies}

The binding specificity of the selected scFvs was determined by ELISA to define the anti-IgM scFv antibodies. Plates were coated with different concentrations of IgM or BSA as a control. All scFvs from positive clones could specifically recognize IgM, but not BSA. The specific binding curve of one scFv, named C1B3, is shown in Figure 3. Compared with the other positive clones, C1B3 could stably express soluble scFv (data not shown) and its binding signal to IgM was the strongest, so it was used to quantify the IgM level in carp serum. The lower limit of grass carp IgM bound by scFv C1B3 was below 25 ng (data not shown). Notably, no $\mathrm{scFv}$ antibodies selected in this study could bind IgM denatured by SDS-PAGE, which meant that they could only recognize the spatial epitope, and not the linear epitope (data not shown).

\subsection{Serum IgM level analysis of grass carp infected with GCRV}

To evaluate the serum IgM levels from both GCRV-infected and uninfected control grass carp, ELISA tests were performed using $\mathrm{scFv} \mathrm{C} 1 \mathrm{~B} 3$ as the primary antibody. As shown in Figure 4A, serum IgM stayed at about the same level in both infected and control grass carp within 3 dpi. However, the level of serum IgM in virus-infected carp began to increase at $4 \mathrm{dpi}$, and was about two times higher than in control carp at 5 and 7 dpi. There was no visible change in serum IgM in control grass carp, indicating that serum IgM was induced to a higher level by GCRV infection. The diseased grass carp presented the typical systemic hemorrhagic phenotype in muscle tissue at 6 or 7 dpi (Figure 4B), and viral factory-like structures and many mature viral particles were also observed by transmission electron microscopy (TEM) of ultrathin-sections (data not shown). No hemorrhagic symptoms or virions were detected in the control carp group (Figure 4C).

\subsection{Antigenic analysis of GCRV structural proteins}

To identify which GCRV structural protein had the strongest antigenicity to induce specific IgM against the virus in grass carp, Western blot assays were performed using antiserum from grass carp infected with GCRV as the primary antibody and $\mathrm{scFv} \mathrm{C} 1 \mathrm{~B} 3$ as the secondary antibody. To conduct the experiment, GCRV particles were purified by 
Table 1 Amino acid sequences of the CDR of $\mathrm{H}$ and $\mathrm{L}$ chain variable regions of positive scFvs clones

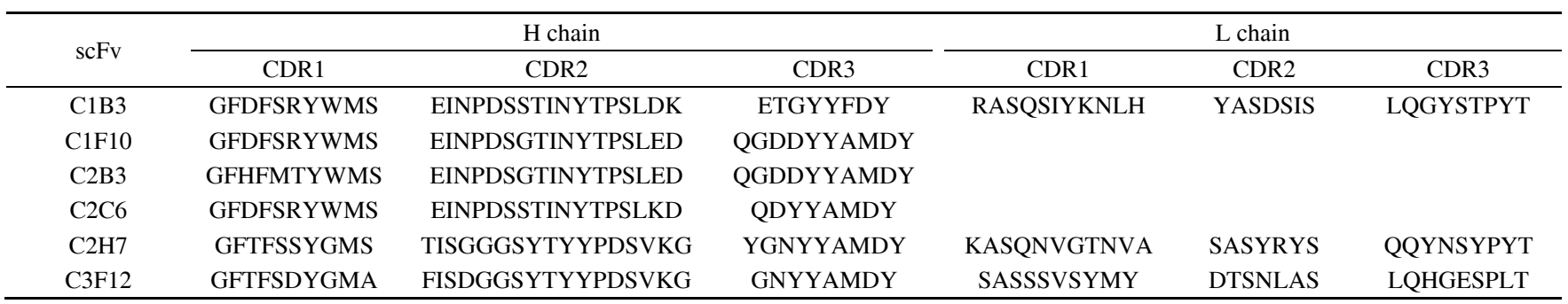

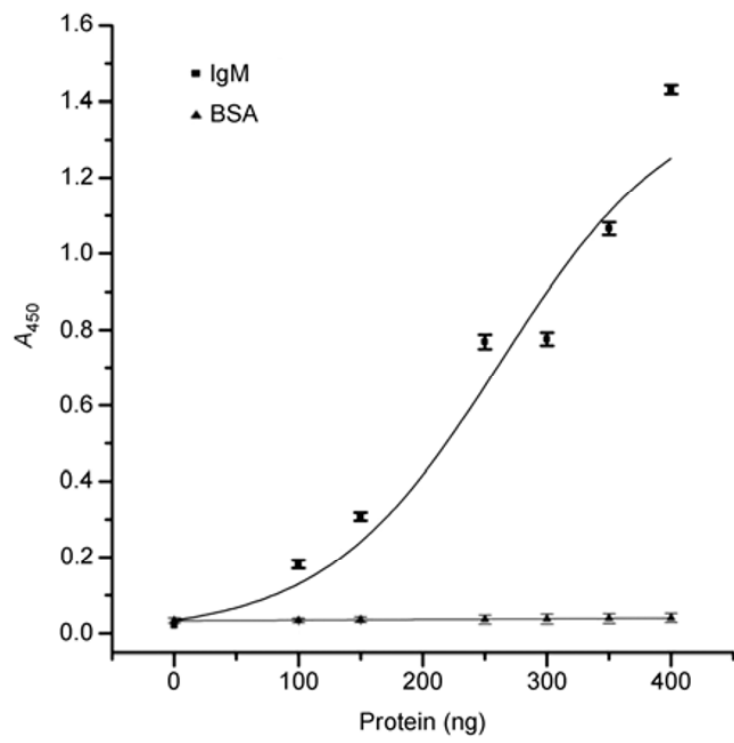

Figure 3 ELISA for binding specificity of scFv C1B3 to IgM from grass carp. Microplates were coated with (-) 100-400 ng/well of purified IgM, or $(\boldsymbol{\Delta})$ the same protein concentration of BSA as a control. Values are mean $\pm \mathrm{SD}$. The asterisks denote significant difference in paired $t$ tests, $n=3$, $P<0.05$.

cesium chloride $(\mathrm{CsCl})$ density gradient centrifugation from infected cell supernatant. The purified intact virions appeared to have an overall double capsid shell about $80 \mathrm{~nm}$ in diameter in TEM, as shown in Figure 5A. The purified virus sample was then analyzed by SDS-PAGE to verify whether the viral structural proteins stayed intact. It appeared, as shown in Figure 5B, that the purified virus sample contained seven capsid proteins VP1-VP7, indicating that the purified GCRV preparation comprised complete structural proteins. Western blot assays were then performed with three repeats using $\mathrm{scFv} \mathrm{C} 1 \mathrm{~B} 3$ as a secondary antibody against IgM. As shown in Figure 5C, the VP7 protein of GCRV, but not the other structural proteins (VP1-VP6), was recognized by serum IgM from GCRVinfected grass carp with a specific blotting band at about 34 $\mathrm{kD}$. There was no specific immuno-blotting band detected at the same position by serum from uninfected control grass carp. Some large molecular blotting bands were observed in sera from both infected and uninfected grass carp, indicating that they were non-specific immuno-cross reaction bands. These results implied that VP7 was the strongest

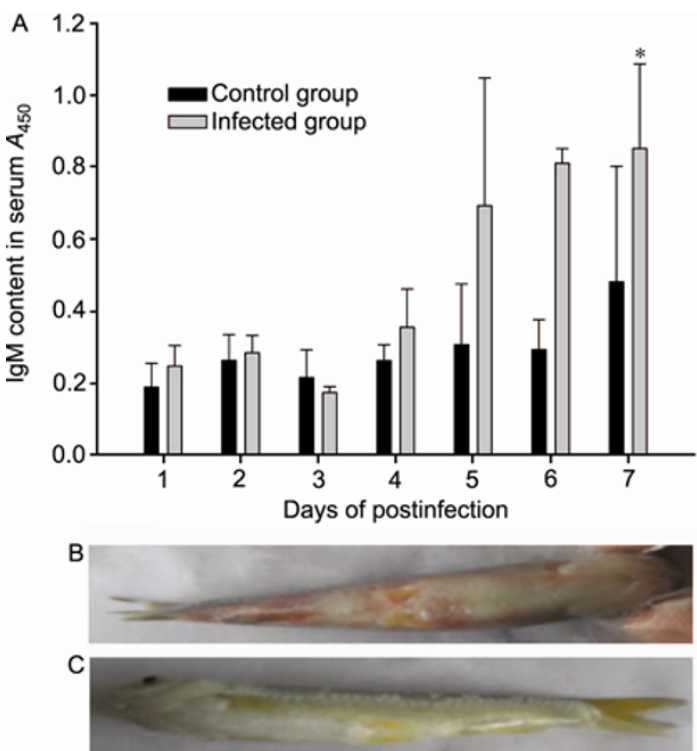

Figure 4 Infected and uninfected fish symptoms and ELISA for changes of IgM level in grass carp serum after GCRV infection. A, A 96-well plate was coated with $1 \mu \mathrm{L}$ of serum from grass carp per well and the IgM level was determined by $\mathrm{scFv} \mathrm{C} 1 \mathrm{~B} 3$ from periplasmic extract with 1:8 dilution. Values are mean $\pm \mathrm{SD}$. The asterisks denote significant difference in paired $t$ tests, $n=3, P<0.05$. B and $\mathrm{C}$, Photographs of typical hemorrhagic diseased grass carp from the GCRV infected group (B) and healthy-looking grass carp from the uninfected control group (C) at 6 dpi.

antigen to induce specific $\operatorname{IgM}$ in grass carp serum.

\section{Discussion}

The immune system plays a critical role in host defense against viral infection. As a major immunoglobulin, IgM mediates humoral adaptive immunity in fish. In this study, an $\mathrm{scFv}$ antibody against $\mathrm{IgM}$ was used as a tool to detect changes in IgM level to measure immune response to GCRV infection in grass carp. Interestingly, when using scFv C1B3 to detect which GCRV viral proteins could be specifically recognized by $\operatorname{IgM}$ from infected carp serum, only VP7 was recognized by IgM from the antiserum. This result suggests that the VP7 protein might be a major antigen for GCRV to induce a stronger immune response in grass carp. In addition, in the Western blot assay some bigger viral protein bands were bound equally by IgM from 
both antiserum and control serum of grass carp (as shown in Figure 5C). This means that some viral proteins can cross-react with some kinds of IgM from grass carp unrelated to the immune response. Because only VP7 can induce higher production of specific $\operatorname{IgM}$, this viral capsid protein should have stronger antigenicity than the other viral structural proteins in grass carp. This result is consistent with our previous investigation [14].

A phage display antibody library provided a plentiful source of monoclonal recombinant antibodies, and made the selection of specific antibody against IgM from grass carp easier than traditional methods for this type of research. These results also reveal that the binding site for $\mathrm{scFv} \mathrm{C} 1 \mathrm{~B} 3$ should be on the constant region of $\operatorname{IgM}$ because this $\mathrm{scFV}$ can recognize any kind of serum IgM with specific or non-specific binding. It is not possible for scFv C1B3 to bind to a variable region of $\operatorname{IgM}$, because these regions are binding domains for the antigen.

Recent studies on the GCRV genome and 3D structure have revealed that the VP5 and VP7 proteins comprise the outer capsid shell of the virus, and resemble the outer capsid proteins $\mu 1$ and $\sigma 3$ of MRV, respectively, such that VP5 and VP7 in GCRV might play critical roles during virus entry into cells [25-27]. VP7, located outward at icosahedral positions through its close interactions with underlying VP5 subunits, is the major surface protein of GCRV virions, and is recognized to provide stability for the virion or VP5 protein [11]. However, GCRV lacks a counterpart to the

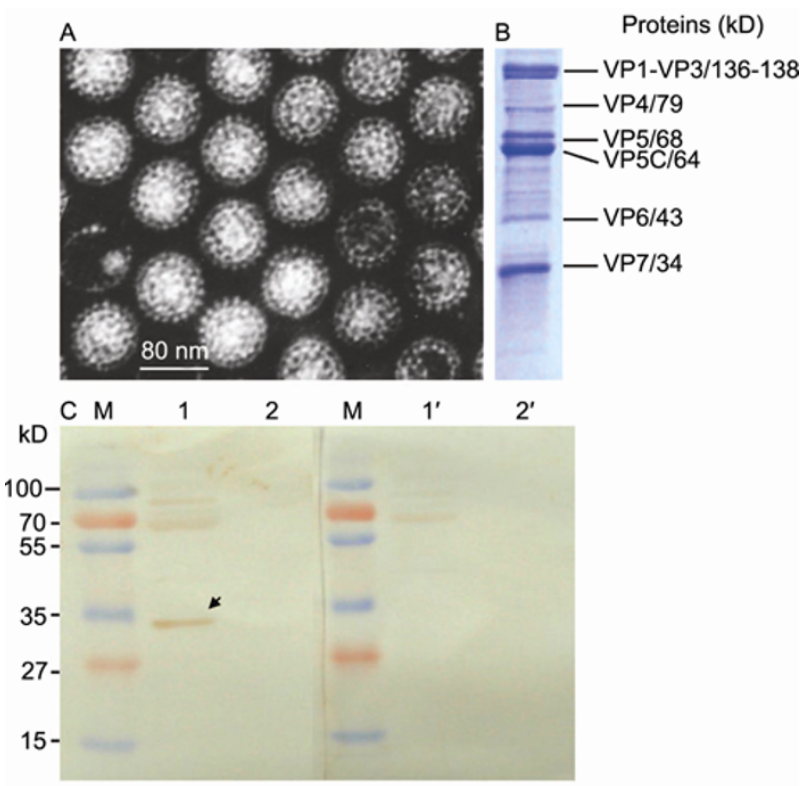

Figure 5 GCRV particles, protein components and Western blotting assay. A, Purified GCRV particles observed by TEM. B, SDS-PAGE analysis of GCRV structural proteins. C, Western blotting assay. Lane M, protein molecular weight markers; lane 1, GCRV reacted with IgM from mixed sera of three GCRV-infected grass carp at 6 dpi detected by $\mathrm{scFv}$ C1B3; lane 2, CIK cells as a control group; lane 1', GCRV reacted with IgM from mixed sera of three uninfected grass carp detected by $\mathrm{scFv}$ C1B3; lane 2', CIK cells as a control group; the arrowhead indicates the VP7 protein band.
MRV $\sigma 1$ protein, which functions as the cell attachment protein situated on each fivefold vertex [11], so the VP7 protein might play a key role in interacting with the host cell during virus infection. An earlier study indicated that the complete digestion of VP7 and partial cleavage of VP5 leads to enhanced infectivity, suggesting that VP7 and VP5 may cooperate with each other for cell entry during viral infection [25]. Moreover, amongst all seven structural proteins of GCRV, the VP7 capsid protein is the most divergent based on genome sequence and single particle Cryo-EM image analyses, suggesting that VP7 is a more specific antigen than the other GCRV proteins.

To evaluate the antigenicity of viral proteins in a fish system with a view to developing an effective recombinant vaccine, we investigated serum IgM level changes for immune response against GCRV in grass carp using an scFv recombinant antibody. Because of the high virulence of GCRV, it is necessary to find a simple and effective way to vaccinate grass carp. Inactive vaccines for GCRV have been developed for many years, but their application is limited because of the high consumption of purified virions or viral antigen preparations. Therefore, it is important to develop a recombinant vaccine to reduce production costs and seek new ways for effective disease prevention. The fish immune system is quite different from the mammalian system; its primary immunoglobulin against pathogens is IgM, not IgG as in mammals. As such, an scFv antibody against $\operatorname{IgM}$ could be applied as a useful tool to select stronger antigenic viral proteins in fish for designing a recombinant vaccine against GCRV or other pathogens.

This work was supported by the National Basic Research Program of China (2009CB118701, 2009CB118704) and the National Natural Science Foundation of China (31072233, 31172434).

1 Jiang Y, Ahne W. Some properties of the etiological agent of the hemorrhagic disease of grass carp and black carp. In: Ahne W, Kurstak E, eds. Viruses of Lower Vertebrates. Berlin: SpringerVerlag, 1989. 227-239

2 Fang Q, Ke L H, Cai Y Q. Growth characterization and high titre culture of GCHV. Virol Sin, 1989, 4: 315-319

3 Ke L H, Fang Q, Cai Y Q. Characteristics of a new isolation of hemorrhagic virus of grass carp. Acta Hydrobiol Sin, 1990, 14: 153-159

4 Zhang L, Luo Q, Fang Q, et al. An improved RT-PCR assay for rapid and sensitive detection of grass carp reovirus. J Virol Methods, 2010, 169: 28-33

5 Ahne W. Viral infectious of aquatic animals with special reference to asian aquaculture. Ann rev Fish Dis, 1994, 4: 375-388

6 Ding Q Q, Yu L F, Wang X L, et al. Study on infecting other fishes with grass carp hemorrhagic virus. Virol Sin, 1991, 6: 371-373

7 Wang T H, Chen H, Chen H. Preliminary studies on the susceptibility of Gobiocypris rarus to hemorrhagic virus of grass carp. Acta Hydrobiol Sin, 1994, 18: 144-149

8 Rangel A A, Rockemann D D, Hetrick F M, et al. Identification of grass carp hemorrhage virus as a new genogroup of Aquareovirus. J Gen Virol, 1999, 80: 2399-2402

9 Fang Q, Attoui H, Francois J, et al. Sequence of genome segments 1, 2 and 3 of the grass carp reovirus (genus Aquareovirus, family Reoviridae). Biochem Biophys Res Comm, 2000, 274: 762-766 
10 Fang Q, Shah S, Liang Y, et al. 3D reconstruction and capsid protein character-ization of grass carp reovirus. Sci China Ser C-Life Sci, 2005, 48: 593-600

11 Cheng L, Fang Q, Shah S, et al. Subnanometer-resolution structures of the grass carp reovirus core and virion. J Mol Biol, 2008, 382: 213-222

12 Cheng L, Zhu J, Hui W H, et al. Backbone model of an aquareovirus virion by cryo-electron microscopy and bioinformatics. J Mol Biol, 2010, 397: 852-863

13 He Y, Xu H, Yang Q, et al. The use of an in vitro microneutralization assay to evaluate the potential of recombinant VP5 protein as an antigen for vaccinating against Grass carp reovirus. Virol J, 2011, 8: 132

14 Shao L, Sun X Y, Fang Q. Antibodies against outer-capsid proteins of Grass carp reovirus expressed in E. coli are capable of neutralizing viral infectivity. Virol J, 2011, 8: 347

15 Zhang L L, Lei C F, Fan C, et al. Expression of outer capsid protein VP5 of Grass carp reovirus in E. coli and analysis of its immunogenicity. Virol Sin, 2009, 24: 545-551

16 Warr G W. The immunoglobulin genes of fish. Dev Comp Immunol, 1995, 19: 1-12

17 Hirono I, Nam B H, Enomoto J, et al. Cloning and characterization of a cDNA encoding Japanese flounder Paralichthys olivaceus IgD. Fish \& Shellfish Immunol, 2003, 15: 63-70

18 Danilova N, Bussmann J, Jekosch K, et al. The immunoglobulin heavy-chain locus in zebrafish: identification and expression of a previously unknown isotype, immunoglobulin Z. Nat Immunol, 2005, 6: 295-302
19 Hansen J D, Landis E D, Phillips R B. Discovery of a unique Ig heavy-chain isotype (IgT) in rainbow trout: implications for a distinctive B cell developmental pathway in teleost fish. Proc Natl Acad Sci USA, 2005, 102: 6919-6924

20 Watts M, Munday B L, Burke C M. Immune responses of teleost fish. Aust Vet J, 2001, 79: 570-574

21 Wilson M R, Warr G W. Fish immunoglobulins and the genes that encode them. Annual Rev Fish Dis, 1992, 2: 201-221

22 Chen C L, Luo S X, Zhang X H, et al. Analysis and characterization of serum immunoglobulin IgM tetramer from grass carp (Ctenopharyngodon idella). Acta Hydrobiol Sin, 2012, 36: 173-176

23 Bradford M M. A rapid and sensitive method for the quantitation of microgram quantities of protein utilizing the principle of protein-dye binding. Anal Biochem, 1976, 72: 248-254

24 Dai H, Gao H, Zhao X, et al. Construction and characterization of a novel recombinant single-chain variable fragment antibody against White spot syndrome virus from shrimp. J Immunol Methods, 2003, 279: 267-275

25 Fang Q, Seng E, Ding Q Q, et al. Characterization of infectious particles of grass carp reovirus by treatment with proteases. Arch Virol, 2008, 153: 675-682

26 Liemann S, Chandran K, Baker T S, et al. Structure of the reovirus membrane penetration protein, $\mu 1$, in a complex with its protector protein, $\sigma 3$. Cell, 2002, 108: 283-295

27 Zhang X, Jin L, Fang Q, et al. 3.3 A cryo-EM structure of a nonenveloped virus reveals a priming mechanism for cell entry. Cell, 2010, 141: 472-482

Open Access This article is distributed under the terms of the Creative Commons Attribution License which permits any use, distribution, and reproduction in any medium, provided the original author(s) and source are credited. 